\title{
NEW INFLATION IN SUPERSYMMETRIC THEORIES
}

\author{
Andreas ALBRECHT ${ }^{1}$, Savas DIMOPOULOS ${ }^{2}$, W. FISCHLER ${ }^{1}$, Edward W. KOLB ${ }^{3}$, \\ Stuart RABY ${ }^{3,4}$, and Paul J. STEINHARDT ${ }^{1}$ \\ 'Department of Physics, University of Pennsylvania, Philadelphia, PA 19174, USA \\ 'Department of Physics, Stanford University, Stanford, CA 94305, USA \\ ${ }^{3}$ Theoretical Division, Los Alamos National Laboratory, Los Alamos, NM 87545, USA \\ ${ }^{4}$ Department of Physics, University of Michigan, Ann Arbor, MI 48109, USA
}

Received 22 March 1983

(Revised 15 August 1983)

\begin{abstract}
A new inflationary universe scenario is considered based upon phase transitions in supersymmetric unified models where the Higgs potential is of the Witten-O'Raifeartaigh type.
\end{abstract}

\section{Introduction}

Although the standard hot-big-bang model [1] provides a remarkably successful description of the large-scale structure of our universe, there are several problems which are not fully understood. The remarkable degree of homogeneity and isotropy observed on large scales is not a consequence of the model; rather it is put in by hand. The observed flatness of the universe is another feature which is not predicted, and in fact is quite surprising [2]. The standard cosmological model predicts that grand unified monopoles, if they exist, should be about as plentiful as nucleons [3]; a prediction that misses by at least fourteen order of magnitude.

Guth [4] made the observation that the above problems could be solved if during the history of the universe a large amount of entropy could be created. In Guth's original proposal, the universe supercools into a metastable phase during a first-order phase transition associated with spontaneous symmetry breaking. During supercooling the vacuum energy density causes a period of de-Sitter expansion [5], and the scale factor of the universe may increase exponentially. If the universe reheats after the transition to a temperature comparable to the temperature before supercooling, a huge amount of entropy may be generated. The phase transition is normally completed when small bubbles of true vacuum nucleate and coalesce. However, detailed analysis [6] has demonstrated that nucleation will not proceed quickly enough to percolate the universe with bubbles of true vacuum and the transition is never completed; hence, the death of the original idea. 
Recently Linde [7] and Albrecht and Steinhardt [8] have proposed a new scenario for inflation. They noticed that for Higgs potentials of the Coleman-Weinberg type [9] as the temperature approaches zero the metastable phase becomes unstable and different regions of the universe begin to evolve toward the stable phase. The Coleman-Weinberg potential is so flat near the origin, that it takes the Higgs field many expansion times to reach the bottom of the potential. During the transition, the universe is dominated by the vacuum energy and the scale factor expands exponentially. In this "new inflation" the universe undergoes sufficient inflation for a single region of evolving stable phase to encompass our entire observable inverse. In this "new inflation" reheating is possible for a wide range of parameters [10], so sufficient entropy can be produced.

Several groups have studied the spectrum of perturbations produced in the new inflation [11,12]. They have found that the perturbations produced are (almost) scale-free, i.e. the amplitude of the perturbations is independent of the wavelength. This is exactly the sort of perturbation spectrum needed in the Zel'dovich pancake theory of galaxy formation. The fact that the scale-free perturbation spectrum is automatic in the new inflation scenario is a very encouraging sign.

However the new inflation is not without problems. First, the grand unified theories (GUTs) cannot be considered realistic because of the hierarchy problem [13]. Second, additional parameters have to be fine-tuned to obtain the ColemanWeinberg potential [8]. Third, although the spectrum of perturbations is scale-free, the magnitude of the perturbations is about $10^{5}$ times too large [11,12].

In this paper we consider the new inflation in supersymmetric GUTs employing the Witten-O'Raifeartaigh $[14,15]$ potential. In this model, a large mass scale is generated from a smaller fundamental scale by the inverse hierarchy mechanism, and it was hoped that this could lead to a natural solution to the hierarchy problem. (Later we will discuss objections which have been raised regarding this mechanism.) The Witten-O'Raifeartaigh Higgs potential used in the inverse hierarchy is very flat; the potential varies only logarithmically with field strength. The fact that the potential is flat even close to the minimum of the potential (the Coleman-Weinberg potential is only flat near the origin) means that inflation will occur for large values of field strength, and the resulting perturbations may be small enough [12]. Therefore, the Witten-O'Raifeartaigh potential appears to subsume all the successes of the new inflation, and solves the new inflation problems of hierarchy and large perturbations.

However, the "newer" inflation has its own problem. Implementation of the Witten-O'Raifeartaigh potential to solve the hierarchy problem requires decoupling of the Higgs field from the light degrees of freedom. This decoupling prevents the universe from reheating, so no baryons are created. In addition, due to decoupling, we find that the transition to a radiation dominated universe is completed only after a long period of "matter" domination by the coherent Higgs field energy. As we discuss in sect. 4 , this leads to an unacceptable cosmology. 
Although it seems hard to avoid decoupling when such potentials are used to solve the hierarchy problem, it may be possible to obtain potentials of similar form but without decoupling. No realistic Witten-O'Raifeartaigh model has been proposed without decoupling. Nevertheless, the cosmological consequences of such a model are sufficiently interesting to warrant study.

In sect. 2 we discuss the potential and some particle physics details. In sect. 3 we discuss the equations of motion for the classical Higgs field. In sect. 4 we give the solutions to the equations of motion for several different cases. Sect. 5 presents our conclusions and the prospectus for supersymmetric cosmology.

\section{The Higgs potential}

We will study inflation in a class of supersymmetric models based on the Witten-O'Raifeartaigh Higgs potential. Supersymmetric unified models are a possible solution to the gauge hierarchy problem. The gauge hierarchy problem has two aspects. First, the weak mass is about $10^{-12}$ smaller than the grand mass. Second, such a large mass difference requires fine-tuning of the parameters of the Higgs potential, since radiative corrections will tend to drag the small scale up to the large scale.

Although the stability of the hierarchy is assured by supersymmetry, it does not explain the small number, $m_{\text {weak }} / m_{\mathrm{GUM}}$. Witten suggested that such a large gauge hierarchy occurs naturally in some theories where supersymmetry is spontaneously broken as in the O'Raifeartaigh model.

Dimopoulos and Raby have constructed a supersymmetric unified model based on the gauge group SU(5) [16], which incorporates the Witten-O'Raifeartaigh potential. In the model the superspace potential is constructed from left-handed chiral superfields $Z_{i}^{j}, A_{i}^{j}$, and $X . Z$ and $A$ are in the adjoint representation of SU(5) and $X$ is an SU(5) singlet. The superfields $X, Z$, and $A$ contain the complex scalars $\phi_{X}, \phi_{Z}$, and $\phi_{A}$. The superspace potential contains the terms

$$
W=\lambda_{1} \operatorname{Tr}\left(Z A^{2}\right)+\lambda_{2} X\left(\operatorname{Tr} A^{2}-M^{2}\right)+\cdots,
$$

where $M$ is some mass characterizing the scale of supersymmetry breaking. The tree-level scalar potential is given in terms of the auxiliary fields, $-F_{i}^{*} \equiv \partial W / \partial i$ :

$$
V_{0}=\left|F_{\mathrm{S}}\right|^{2}+\sum\left|F_{Z}\right|^{2}+\sum\left|F_{A}\right|^{2}+\cdots
$$

Since $F_{X}=0, F_{Z}=0$ are inconsistent, supersymmetry is broken at the tree level. Minimization of the potential results in a vacuum expectation value for the scalar fields

$$
\begin{aligned}
& \left\langle\phi_{A}\right\rangle=A_{0} \operatorname{diag}(2,2,2,-3,-3), \\
& \left\langle\phi_{Z}\right\rangle=Z_{0} \operatorname{diag}(2,2,2,-3,-3), \\
& \left\langle\phi_{X}\right\rangle=X_{0},
\end{aligned}
$$


where

$$
A_{0}=\frac{\lambda_{2} M}{\left(\lambda_{1}^{2}+30 \lambda_{2}^{2}\right)^{1 / 2}}, \quad Z_{0}=\frac{\lambda_{2}}{\lambda_{1}} X_{0}
$$

Note that $Z_{0}$ is undetermined at the tree level. From (2.3) it is seen that $S U(5)$ is broken to $\mathrm{SU}(3) \times \mathrm{SU}(2) \times \mathrm{U}(1)$ at the scale $\mu \simeq A_{0}$. However the $\mathrm{SU}(5)$ breaking is not completed at this scale. A scalar field $\tilde{\phi}$, which is a linear combination of SU(5) singlet and adjoint fields, is driven by $\left\langle\phi_{A}\right\rangle$ to obtain an expectation value of the form

$$
\langle\tilde{\phi}\rangle=\phi \operatorname{diag}(3,3,3,-2,-2)
$$

At the tree level, $V(\phi) \simeq \mu^{4}$, the inclusion of one-loop corrections give (for $\phi \gg \mu$ )

$$
V(\phi) \simeq \mu^{4}\left[1+\tilde{b} \ln \frac{\phi}{\mu}\right]+\mathrm{O}(\mu / \phi) .
$$

At the one-loop level, $\tilde{b}$ is a constant proportional to $29 \lambda_{1}^{2}-50 g^{2}$. If $50 g^{2}>29 \lambda_{1}^{2}$ the potential will be minimized for $\phi \gg \mu$. Higher-order effects will result in a $\phi$ dependence of $b$, since the coupling constants will depend (logarithmically) on $\phi$. It is reasonable to expect that asymptotic freedom will force $29 \lambda_{1}^{2}-50 g^{2}$ to change sign at some large value of $\phi$, producing a minimum at $\phi=m_{\mathrm{GUM}}$. Therefore the potential will be of the form

$$
V(\phi)=\mu^{4}\left[1+b \ln ^{2}\left(\phi / m_{\mathrm{GUM}}\right)\right],
$$

where $b$ is a constant (independent of $\phi$ ). We must add or subtract a constant term to make the potential vanish at $m_{\mathrm{GUM}}$. Therefore the potential we will assume will be of the form

$$
V(\phi)=b \mu^{4} \ln ^{2}\left(\phi / m_{G U M}\right),
$$

and we expect $m_{\mathrm{GUM}}$ to be $\mathrm{O}\left(m_{\mathrm{P}}\right)$, where $m_{\mathrm{P}}$ is the Planck mass.

Two related features are necessary to make this potential into a realistic model. First, the supersymmetry breaking scale $\mu$ must be intermediate between the weak scale and the grand scale

$$
\mu=\left(m_{\text {weak }} m_{\mathrm{GUM}}\right)^{1 / 2} \simeq 10^{12} \mathrm{GeV},
$$

(hence geometric hierarchy), and second, the $\phi$ field must couple to light fields only through an intermediate state with mass $m_{\mathrm{GUM}}$. This decoupling is necessary to prevent the weak scale from being dragged up to the intermediate scale of supersym- 
metry breaking. The basic result of decoupling is that the coupling of the $\phi$ field to a state of mass $m_{\mathrm{S}}$ is proportional to $m_{\mathrm{S}}^{2} / m_{\mathrm{GUM}}^{2}$.

Since beginning our investigation, several criticisms have been raised regarding the naturalness of these models. Hall and Hinchliffe [17] have pointed out that it is hard to generate a hierarchy of $m_{\mathrm{GUM}} \gg \mu$ using the O'Raifeartaigh-Witten mechanism. The difficulty comes in arranging for the coefficient of the $\ln \phi$ terms ( $\tilde{b}$ in eq. (2.6)) in the effective potential to change sign and create a minimum at a large enough GUT scale. To do this the various couplings must have appropriate values at the $\mu$ scale so that when they are evolved according to the renormalization group equations they meet the condition that $\tilde{b}$ change sign at a large scale. Examination of the renormalization group equations indicates that only a very small class of choices of couplings cause $\tilde{b}$ to change sign at a sufficiently large scale. In fact, to achieve the required relationship $m_{G U M}=\mathrm{O}\left(\mu \times 10^{7}\right)$ the values of the couplings must be restricted to a region $10^{-6}$ the size of the allowed region in parameter space.

This picture changes, however, when attempts are made to incorporate gravity into the picture. For example, Ovrut and Raby [18] have coupled this model to $N=1$ supergravity and have found in this case that there is a large region of parameter space where reasonable hierarchies result. This is because many choices of parameters which were originally rejected on the grounds that they corresponded to a potential with no minimum are now rendered acceptable. The gravitational corrections produce a minimum close to the Planck scale in these potentials. (We have not gained so much as far as the hierarchy problem is concerned, because the Planck scale has been introduced by hand; however, we can consider these as effective potentials and study their impact on inflation.)

We have investigated the cosmology of models with gravitational corrections included and found no significant changes from our results using this simpler potential (eq. (2.6)). It should be pointed out that achieving sufficient inflation is, however, made a little easier in these models because there is somewhat faster expansion rate and a longer inflation epoch. However, decoupling which plagues the simpler model (eq. (2.6)) plagues these models too. Since our work on the estimated effects of gravity has been reported in more detail elsewhere [19], we will only discuss the simpler potential described by eq. (2.7).

The other naturalness problem arises when one tries to arrange a sensible low-energy phenomenology [20-23]. The Higgs doublets can be made light only at the cost of fine-tuning (thus removing the alleged lack of fine-tuning as a major motivation for building these models).

\section{The equations of motion for $\phi$}

At high temperatures, the universe should have been in the symmetric $\phi=0$ state [24]. The usual scenario for spontaneous symmetry breaking in the early universe is that the system remains in the symmetric phase until the temperature reaches a 
critical temperature, $T_{c}$, of the order of the vacuum expectation value, $\langle\phi\rangle$. An interesting phenomenon in the model of the last section is that the critical temperature, $T_{\mathrm{c}}$, is $\mathrm{O}(\mu)$; much less than $\langle\phi\rangle$, which is $\mathrm{O}\left(m_{\mathrm{p}}\right)$ [21]. At some $T=T_{\mathrm{c}}$, with $\mu^{2} / m_{\mathrm{P}} \leqslant T_{\mathrm{c}} \leqslant \mu$, the transition occurs either via thermal or quantum fluctuations [21]. (Note the lower limit governed by the Hawking temperature.) Immediately after the transition,

$$
\phi \simeq \mu, \quad \dot{\phi} \simeq 0,
$$

and $\phi$ is roughly uniform over a coherence length of order $\mu^{-1}$. (Even if $\dot{\phi} \simeq \mu^{2}$, our results will not be affected.) The transition is not completed until the coherent field $\phi$ travels down the logarithmic potential to the minimum $\phi=m_{\mathrm{GUM}}$. During this time, $V(\phi)$ dominates the radiation energy density and the universe is in a de Sitter phase [5].

In order to write the equations of motion for the universe we must differentiate between three types of energy density as distinguished by their equation of state at $T \simeq \mu$ : (i) $\rho_{\mathrm{r}}$, the radiation energy density, with equation of state $p_{\mathrm{r}}=\frac{1}{3} \rho_{R}$; (ii) $\rho_{m}$, the energy density for states of mass $m=\mu+\phi$, with equation of state $p_{m}=0$ (these states include the gauge states in the coset space $\mathrm{SU}(5) / \mathrm{SU}(3) \times \mathrm{SU}(2) \times \mathrm{U}(1)$, the $A$ states, and monopoles). Some of the energy from the coherent $\phi$ field is required to give mass as well as kinetic energy to these particles. (iii) $\rho_{\phi}$, the energy density in the coherent $\phi$ field given by $\rho_{\phi}=\frac{1}{2} \dot{\phi}^{2}+V(\phi)$ with an equation of state that results in $p_{\phi}=\frac{1}{2} \dot{\phi}^{2}-V(\phi)$. The equations of motion are

$$
\begin{aligned}
\ddot{\phi} & =-\frac{\mathrm{d} V}{\mathrm{~d} \phi}-3 \frac{\dot{R}}{R} \dot{\phi}-\Gamma \dot{\phi}-\frac{\rho_{m}}{m}, \\
\dot{\rho}_{\mathrm{r}} & =-4 \frac{\dot{R}}{R} \rho_{\mathrm{r}}+\Gamma \dot{\phi}^{2}+\Gamma_{m}\left(\rho_{m}-\rho_{m}^{\mathrm{eq}}\right), \\
\dot{\rho}_{m} & =-3 \frac{\dot{R}}{R} \rho_{m}+\frac{\dot{\phi}}{m} \rho_{m}-\Gamma_{m}\left(\rho_{m}-\rho_{m}^{\mathrm{eq}}\right), \\
\left(\frac{\dot{R}}{R}\right)^{2} & =\frac{8 \pi}{3 m_{\mathrm{P}}^{2}}\left[\rho_{\mathrm{r}}+\rho_{m}+\rho_{\phi}\right],
\end{aligned}
$$

where $\dot{R} / R$ is the expansion rate of the universe, $m=\mu+\phi, \rho_{m}^{\mathrm{eq}}=(m T / 2 \pi)^{3 / 2} \mathrm{e}^{-m / T}$, $\rho_{\mathrm{r}}=\frac{1}{15} \pi^{2} T^{4}$, and $\Gamma_{m}=\alpha m$, where $\alpha$ is the square of a coupling constant.

Some of the terms in (3.2) require explanations: $\Gamma$ is the rate for the coherent state $\phi$ to transfer energy into radiation. As discussed by Abbott, Farhi, and Wise [26], this is simply the decay rate for the scalar field $\phi$, with mass $m_{\phi} \simeq \mu^{2} / m_{\mathrm{P}}$, to decay into radiation. The term $\rho_{m} / m$ takes into account the transfer of energy from the 
coherent field $\phi$ to those states with mass $m . \Gamma_{m}$ is the decay rate for those states of mass $m$. (For monopoles, $\Gamma_{m}=0$.)

The next section will discuss solutions to (3.2) with initial conditions

$$
\dot{\phi}=0, \quad \phi \simeq \mu, \quad \rho_{m} \simeq \rho_{\mathrm{r}} \simeq \mu^{4} .
$$

However, before discussing the solutions to (3.2), we will discuss how decoupling enters the problem.

Decoupling is important in the calculation of $\Gamma$. If the field $\phi$ couples to some state (with mass less than $m_{\phi}$ ) with a coupling constant $h$, then the decay rate is given by

$$
\Gamma \simeq h^{2} m_{\phi}
$$

where the mass $m_{\phi}$ is given by the curvature of the potential,

$$
m_{\phi}^{2}=\frac{\mathrm{d}^{2} V}{\mathrm{~d} \phi^{2}} \simeq \frac{2 b \mu^{4}}{\phi^{2}} .
$$

As discussed in sect. 2, the coupling of $\phi$ to a state of mass $m_{\mathrm{S}}$ is suppressed by $\left(m_{\mathrm{S}} / m_{\mathrm{GUM}}\right)$. Therefore, since $m_{\mathrm{GUM}}=\phi$, the largest $h$ is of order $\left(m_{\phi} / \phi\right)$ and the decay width is

$$
\begin{aligned}
\Gamma & \simeq \frac{m_{\phi}^{3}}{\phi^{2}} \\
& =\kappa \frac{\mu^{6}}{\phi^{5}},
\end{aligned}
$$

where $\kappa$ is a number of order unity.

\section{The development of the phase transition}

Before giving the numerical results, it is useful to consider some analytic arguments. Because of decoupling, $\Gamma=0$ is a good approximation. We also find that the initial $\rho_{m}$ is rapidly redshifted away and is not important in the equation for $\ddot{\phi}$. We also find that the system rapidly approaches a "terminal" velocity, in the sense that $\ddot{\phi}=0$ is a good approximation. Therefore, to a reasonable approximation the system (3.2) can be described by

$$
-\frac{\mathrm{d} V}{\mathrm{~d} \phi}-3 \frac{\dot{R}}{R} \dot{\phi}=0, \quad \dot{\rho}_{\mathrm{r}}=-4 \frac{\dot{R}}{R} \rho_{\mathrm{r}}
$$


If we further assume that

$$
\left(\frac{\dot{R}}{R}\right)^{2}=\frac{8 \pi}{3 m_{\mathrm{P}}^{2}} V(\phi),
$$

the equation for $\phi$ becomes (with $V(\phi)$ defined in (2.8))

$$
3\left(\frac{8}{3} \pi\right)^{1 / 2} \frac{\dot{\phi}}{m_{\mathrm{P}}}=2 b^{1 / 2} \mu^{2} / \phi .
$$

During the de Sitter phase, the scale factor $R$ is given in terms of the scale factor at the beginning of inflation, $R_{0}$, by

$$
\ln \frac{R}{R_{0}}=\int \frac{\dot{R}}{R} \mathrm{~d} t=\int_{\phi \simeq 0}^{\phi=m_{\mathrm{GUM}}} \frac{\dot{R}}{R} \dot{\phi}^{-1} \mathrm{~d} \phi .
$$

Using (4.3) for $\dot{\phi}$, we find

$$
\ln \frac{R}{R_{0}}=\pi\left(\frac{m_{\mathrm{GUM}}}{m_{\mathrm{P}}}\right)^{2}
$$

Note that this answer is independent of $\mu$ and $b$. This result suggests that for sufficient inflation, the grand scale must be 4 or 5 times larger than the Planck mass (in which case quantum-gravitational effects may invalidate our analysis). Numerical integration of the system is in very good agreement with (4.5).

We must emphasize that even though the universe undergoes sufficient exponential expansion, a successful inflationary universe scenario requires not just exponential expansion, but also the subsequent creation of entropy. If $\Gamma \approx 0$, then the vacuum energy is not pumped into radiation and the expansion is adiabatic: no entropy is created.

Moreover, since the energy originally in the coherent Higgs field is not transferred to radiation, it is decreased only by the redshift of the "momentum" of the Higgs field. This decreases $\rho_{\phi}$ as $3 \dot{R} / R$ (like a massive particle), compared to the $4 \dot{R} / R$ redshift of the radiation energy density. Hence, $\rho_{\phi}$ decreases more slowly than $\rho_{\mathrm{r}}$, and will dominate until $\phi$ decay.

Although $\Gamma=0$ is a good approximation during the development of the phase transition, eventually $\Gamma$ becomes comparable to the expansion rate, and the $\phi$-field "decays" [27, 28], creating entropy. The expansion rate during the $\phi$ matter dominated era is

$$
H^{2}(T)=\frac{8}{3} \pi \frac{\rho_{\phi}}{m_{\mathrm{P}}^{2}}=\left(\frac{8}{3} \pi\right) \rho_{\phi}\left(T_{\mathrm{M}}\right)\left(\frac{T}{T_{\mathrm{M}}}\right)^{3} m_{\mathrm{P}}^{-2},
$$


where $\rho_{\phi}\left(T_{\mathrm{M}}\right)$ is the energy density in the $\phi$ field when $\phi$ first reaches the minimum of the potential and starts oscillating about the minimum. Equating $H_{\phi}$ and $\Gamma$, we find the temperature of the universe at $\phi$ decay to be

$$
T_{\mathrm{D}}^{3}=\left(\frac{8}{3} \pi\right)^{-1} \frac{\mu^{12} m_{\mathrm{P}}^{2} T_{\mathrm{M}}^{3}}{m_{\mathrm{GUM}}^{10} \rho_{\phi}\left(T_{\mathrm{M}}\right)} .
$$

Assuming the decay products of the $\phi$ field are instantly thermalized, the temperature of the universe after decay is given by $\left(H_{\phi}\left(T_{\mathrm{D}}\right) \equiv \Gamma\right)$

$$
\begin{aligned}
T_{\mathrm{A}}^{4} & =\rho_{\phi}\left(T_{\mathrm{D}}\right)=H_{\phi}^{2}\left(T_{\mathrm{D}}\right) m_{\mathrm{P}}^{2}\left(\frac{8}{3} \pi\right)^{-1} \\
& =\left(\frac{3}{8} \pi\right) \mu^{12} m_{\mathrm{P}}^{2} / m_{\mathrm{GUM}}^{10} .
\end{aligned}
$$

The entropy density of the universe is increased by a factor

$$
\Delta=\left(\frac{T_{\mathrm{A}}}{T_{\mathrm{D}}}\right)^{3}=\left(\frac{8}{3} \pi\right)^{1 / 4} \frac{\rho_{\phi}\left(T_{\mathrm{M}}\right) m_{\mathrm{GUM}}^{5 / 2}}{\mu^{3} m_{\mathrm{P}}^{1 / 2} T_{\mathrm{M}}^{3}} .
$$

As mentioned above $T_{\mathrm{M}} \sim \mu \exp \left(-\pi m_{\mathrm{GUM}}^{2} / m_{\mathrm{P}}^{2}\right)$. The value of $\rho_{\phi}\left(T_{\mathrm{M}}\right)$ is not as simple to estimate. Numerically, we have found that from $\phi \simeq 0$ until $\phi$ starts oscillation about the minimum, $\rho_{\phi} \sim T^{3}$. Therefore $\rho_{\phi}\left(T_{\mathrm{M}}\right) \sim \rho_{\phi}(0)\left(T_{\mathrm{M}} / T_{0}\right)^{3} \sim \mu T_{\mathrm{M}}^{3}$, where $\rho_{\phi}(0) \simeq \mu^{4}$ is the initial energy density in the Higgs field, and $T_{0}$ is the initial temperature at the start of the transition $\left(T_{0} \sim \mu\right)$. The entropy is therefore increased by a factor

$$
\Delta \simeq m_{\mathrm{GUM}}^{\mathrm{S} / 2} / \mu^{2} m_{\mathrm{P}}^{1 / 2} \simeq 10^{14},
$$

for $m_{\mathrm{GUM}}=m_{\mathrm{P}}$, and $\mu=10^{12} \mathrm{GeV}$.

This increase in entropy is too small to be of any consequence in solving the cosmological problems discussed in the introduction. Since the temperature of the universe after decay is much lower than the masses of particles whose out-of-equilibrium decay would be responsible for baryogenesis, the usual explanation for the baryon asymmetry of the universe does not apply. (Some baryon asymmetry can in principle be generated in the decay of $\phi$ itself, but the baryon number violation in $\phi$ decay is suppressed by $\Delta$.) Even excluding the baryogenesis problem, the reheating temperature is at best marginal for nucleosynthesis. We conclude that this scenario is not the solution to any problems, and indeed may result in more problems.

Although we have demonstrated that sufficient exponential expansion may result, decoupling prevents the transition from ever terminating. This problem, however, is not inherent in the Higgs potential, but is a result of decoupling. Since the potential rewards are so tempting, we will study inflation with the Witten-O'Raifeartaigh 
potential without decoupling and with a minimum amount of decoupling. For further analysis we will assume $m_{\mathrm{GUM}}=m_{\mathrm{P}}$, and $\mu=10^{12} \mathrm{GeV}$.

First, we consider the case where the decay width is proportional to $\phi$

$$
\Gamma=\kappa \phi,
$$

where again, $\kappa$ is a number of order unity. For $\phi<m_{\mathrm{P}}$, neglecting the $\rho_{m}$ term, the equation for $\ddot{\phi}$ is given by

$$
\ddot{\phi} \simeq b \mu^{4} / \phi-3(\dot{R} / R) \dot{\phi}-\kappa \dot{\phi} \phi,
$$

where we have ignored the $\log$ contribution to $\mathrm{d} V / \mathrm{d} \phi$. Since $\dot{R} / R \sim \mu^{2} / m_{\mathrm{P}} \ll \kappa \phi$, the expression will be controlled by the friction term. Again setting $\ddot{\phi}=0,(4.7)$ becomes

$$
\dot{\phi} \phi^{2} \sim b \mu^{4} \kappa^{-1}, \quad \frac{1}{3}\left(\phi^{3}\right)^{\cdot} \sim b \mu^{4} \kappa^{-1},
$$

and the time to reach $\phi=m_{\mathrm{P}}$ is given by

$$
\Delta t=m_{\mathrm{P}}^{3} \mu^{-4}=\left(\frac{m_{\mathrm{P}}}{\mu}\right)^{2}\left(\frac{\dot{R}}{R}\right)^{-1},
$$

with $\kappa=b=1$. In this case the universe expands by a factor

$$
R / R_{0} \simeq \exp \left(\frac{\dot{R}}{R} \Delta t\right)=\exp \left(m_{\mathrm{p}}^{2} / \mu^{2}\right) \simeq \exp \left(10^{14}\right) .
$$

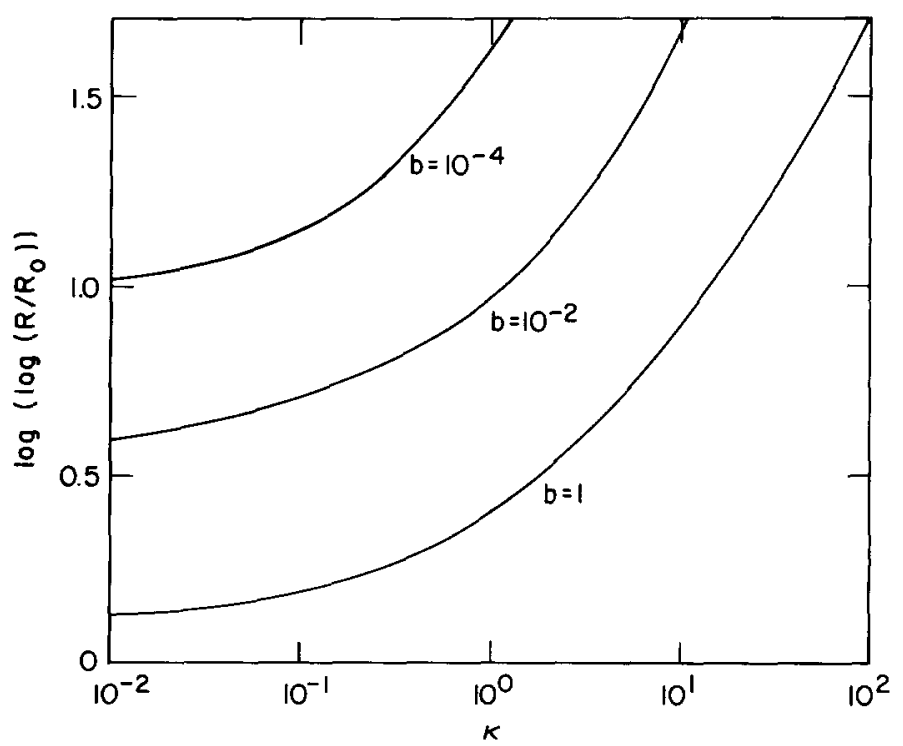

Fig. 1. The amount of inflation during the de-Sitter phase assuming $\Gamma=\kappa \mu^{2} / \phi$. The parameter $b$ appears in the potential. We have used $m_{\mathrm{GUM}}=m_{\mathrm{P}}$ and $\mu=10^{12} \mathrm{GeV}$. 
However, the very large size of $\Gamma$ which causes so much expansion also ruins the prospects for reheating. Numerical analysis shows the $\Gamma \dot{\phi}$ friction term slows the motion of $\phi$ so much that the radiation is always redshifted away faster than it is produced, and no reheating occurs. It is, in any case, unlikely that a model without decoupling will result in a potential that depends logarithmically on $\phi$ as we have assumed here.

As a more likely possibility we consider the Witten-O'Raifeartaigh potential with a minimum amount of decoupling, i.e.,

$$
\Gamma=\kappa m_{\phi}=\kappa \mu^{2} / \phi
$$

We have numerically evaluated the equations of motion with this value of $\Gamma$. This coupling of $\phi$ to radiation does ensure that the radiation energy density will dominate over the energy density of the Higgs field when $\phi$ reaches the minimum of the potential. The final value of $R / R_{0}$ as a function of $\kappa$ for several values of $b$ is shown in fig. 1. For sufficient inflation to occur, $R / R_{0}$ must be about $10^{28}$, or $\log \left(\log R / R_{0}\right) \simeq 1.45$. As seen in fig. 1 , sufficient inflation may occur for reasonable

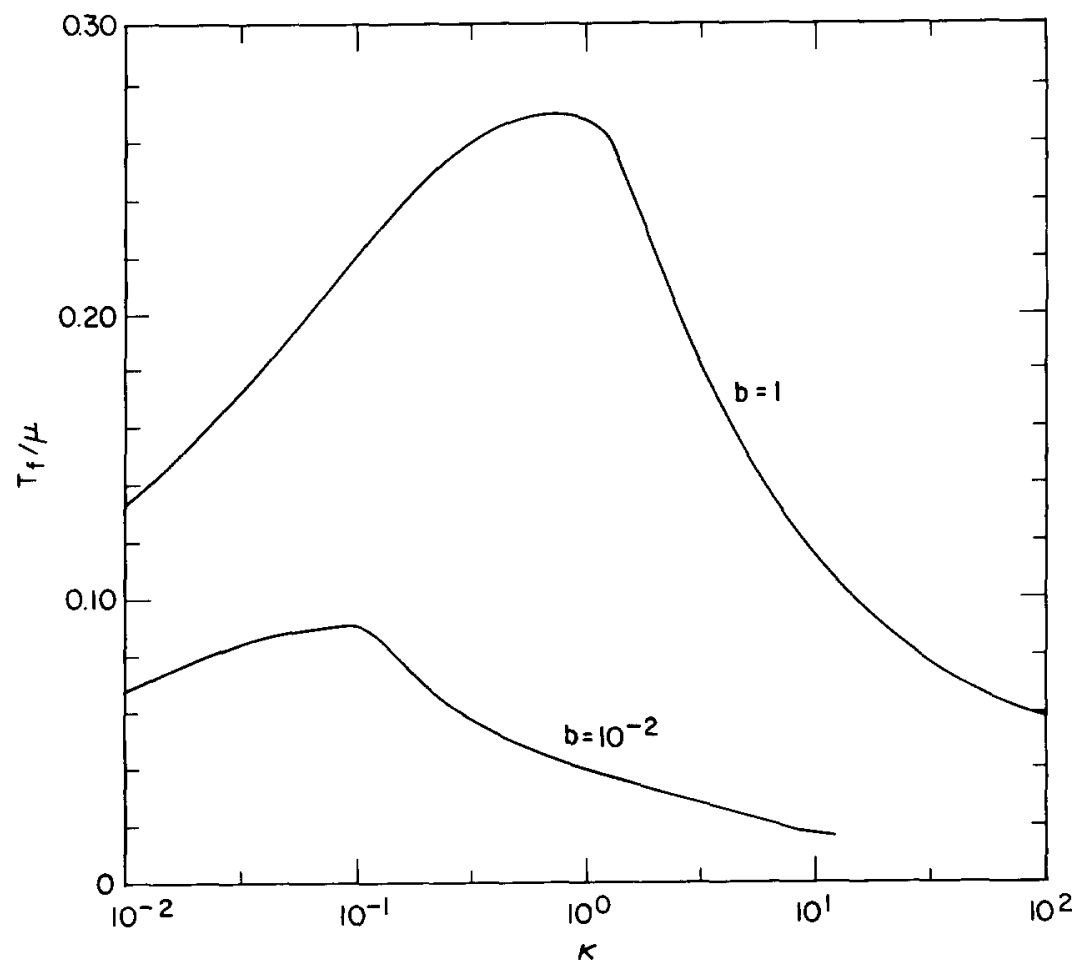

Fig. 2. The final temperature as a function of $\kappa$ if $\Gamma=\kappa \mu^{2} / \phi$ for two values of $b$. We have used $m_{\mathrm{GUM}}=m_{\mathrm{P}}$ and $\mu=10^{12} \mathrm{GeV}$. 
value of $b$ and $\kappa$. We have also calculated the temperature to which the universe reheats. In fig. 2 we give the final temperature as a function of $\kappa$ for $b=1$ and $b=10^{-2}$. Since the number of baryon-number violation bosons with mass $\mu$ is suppressed by the Boltzmann factor $\exp \left(-\mu / T_{\mathrm{f}}\right)$, a final temperature greater than about $0.05 \mu$ is necessary for baryon number generation. As seen from two figures, the parameters may be chosen to result in sufficient inflation, reheating, and baryon-number generation. Therefore, with decoupling as in (4.16), the cosmology can work.

\section{Conclusions}

Supersymmetric unified models are attractive from a particle physics viewpoint. They are also attractive from a cosmological viewpoint because they naturally have a flat potential which is required for "new inflation." The Witten-O'Raifeartaigh potential is flat for large values of the Higgs field (cf. the Coleman-Weinberg potential which is only flat near the origin), which causes inflation for large values of the Higgs field. This not only subsumes the successes of "new inflation" based on the Coleman-Weinberg potential, but may ameliorate the new inflation problem of the size of the density fluctuations induced during the transition. Therefore supersymmetric GUTs offers real hope for a solution to the isotropy, homogeneity, flatness, and monopole problems which are not addressed in the standard cosmology. In addition, the inflation may naturally generate the metric fluctuations needed for galaxy formation.

Although the Witten-O'Raifeartaigh potential is a "potential" success in cosmology, realistic models implementing it seem to require decoupling, which is a potential disaster. The decoupling means that the coherent Higgs field is very inefficient in converting its potential energy to radiation. The resulting universe has a negligible baryon asymmetry, and does not give the standard results for nucleosynthesis.

We have studied the Witten-O'Raifeartaigh potential with a minimum amount of decoupling, and found an acceptable cosmology.

Whether supersymmetric inflation can provide answers to outstanding cosmological problems awaits further study. In this paper we have discussed the problems and the benefits.

Some of these results were reported earlier by us [19].

This work was supported in part by the Department of Energy at Los Alamos and the University of Pennsylvania (EY-76-C-02-3071).

\section{References}

[1] S. Weinberg, Gravitation and cosmology (Wiley, New York, 1973)

[2] R.H. Dicke and P.J.E. Peebles, General relativity: an Einstein centenary survey, eds. S.W. Hawking and W. Israel (Cambridge University Press, London, 1979) 
[3] J. Preskill, Phys. Rev. Lett. 43 (1979) 1365

[4] A.H. Guth, Phys. Rev. D23 (1981) 347

[5] E.W. Kolb and S. Wolfram, App. J. 239 (1980) 428

[6] A.H. Guth and E.J. Weinberg, Nucl. Phys. B212 (1983) 321; S.W. Hawking, I.G. Moss and J.M. Stewart, Phys. Rev. D26 (1982) 2681

[7] A. Linde, Phys. Lett. 108B (1982) 389

[8] A. Albrecht and P.J. Steinhardt, Phys. Rev. Lett. 48 (1982) 1220

[9] S. Coleman and E.J. Weinberg, Phys. Rev. D17 (1973) 1888

[10] A. Albrecht, P.J. Steinhardt, M.S. Turner and F. Wilczek, Phys. Rev. Lett. 48 (1982) 1437

[11] A.H. Guth and S.-Y. Pi, Phys. Rev. Lett. 49 (1982) 1110; S.W. Hawking, Phys. Lett. 115B (1982) 295;

A. Starobinsky, in The very early universe, eds. G. Gibbons, S. Hawking and S. Siklos (Cambridge University Press, London, 1983)

[12] J.M. Bardeen, P.J. Steinhardt, and M.S. Turner, Phys. Rev. D28 (1983) 679

[13] K.G. Wilson, Phys. Rev. D3 (1971) 1818;

L. Susskind, Phys. Rev. D20 (1979) 2619

[14] E. Witten, Phys. Lett. 105B (1981) 267

[15] L. O'Raifeartaigh, Nucl. Phys. B96 (1975) 331

[16] S. Dimopoulos and S. Raby, Nucl. Phys. B 219 (1983) 479

[17] L. Hall and I. Hinchliffe, Phys. Lett. 119B (1982) 128;

M. Einhorn and D.R.T. Jones, Nucl. Phys. B211 (1983) 29;

T. Banks and V. Kaplunovsky, Nucl. Phys. B211 (1983) 529

[18] B. Ovrut and S. Raby, Phys. Lett. 121B (1983) 381

[19] P.J. Steinhardt, in The very early universe, eds. G. Gibbons, S. Hawking, and S. Siklos (Cambridge University Press, London, 1983);

A. Albrecht, S. Dimopoulos, W. Fischler, E.W. Kolb, S. Raby and P.J. Steinhardt, Proc. 3rd Marcel Grossman Meeting on the recent developments of general relativity, Los Alamos National Laboratory Report LA-UR 82-2947

[20] M. Dine and W. Fischler, Nucl. Phys. B204 (1982) 346

[21] T. Banks and V. Kaplunovsky, Nucl. Phys. B211 (1913) 529

[22] J. Polchinski and L. Susskind, Phys. Rev. D26 (1982) 3661

[23] M. Dine and W. Fischler, Pennsylvania Preprint (1982)

[24] D.A. Kirzhnits and A. Linde, ZhETF 67 (1974) 1263

[25] S.-Y. Pi, Phys. Lett. 112B (1982) 441;

P. Ginsparg, Phys. Lett. 112B (1982) 45

[26] L.F. Abbott, E. Farhi and M.B. Wise, Phys. Lett. 117B (1982) 29

[27] A. Albrecht and P. Steinhardt, Phys. Lett. B., to be published;

G.D. Coughlan, W. Fischler, E. W. Kolb, S. Raby and G.G. Ross, Phys. Lett. B., to be published 